INPLASY

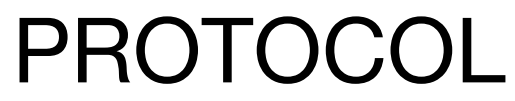

To cite: Ramírez-Vélez et al.

Physical activity and

myosteatosis in adults: a

systematic review and meta-

analysis of randomized

controlled trials. Inplasy

protocol 202070060. doi:

10.37766/inplasy2020.7.0060

Received: 15 July 2020

Published: 15 July 2020

Corresponding author:

Antonio García-Hermoso

antonio.garcia.h@usach.cl

Author Affiliation:

Laboratorio de Ciencias de la

Actividad Física, el Deporte y

la Salud, Universidad de

Santiago de Chile

Support: None.

Review Stage at time of this submission: The review has not yet started.

Conflicts of interest:

None.

\section{Physical activity and myosteatosis in adults: a systematic review and meta-analysis of randomized controlled trials}

Ramírez-Vélez, R; Ezzatvar, Y²; Izquierdo, M³; García-Hermoso, A4 .

Review question / Objective: To estimate the effects of randomized controlled trials using physical activity interventions on myosteatosis (i.e., intermuscular adipose tissue and muscle attenuation).

Condition being studied: Participants over the age of 18 years. Information sources: Two authors independently will search MEDLINE, EMBASE and SPORTDiscus electronic databases.

INPLASY registration number: This protocol was registered with the International Platform of Registered Systematic Review and Meta-Analysis Protocols (INPLASY) on 15 July 2020 and was last updated on 15 July 2020 (registration number INPLASY202070060).

\section{INTRODUCTION}

Review question / Objective: To estimate the effects of randomized controlled trials using physical activity interventions on myosteatosis (i.e., intermuscular adipose tissue and muscle attenuation).
Condition being studied: Participants over the age of 18 years.

\section{METHODS}

Search strategy: The following search terms will be use: 'intermuscular adipose tissue', 'muscle attenuation', 'muscle composition', 'muscle fat infiltration', 'fat 
deposition', 'myosteatosis', 'intramuscular fat', 'fatty infiltration', 'exercise', 'physical activity, 'weight loss', 'resistance exercise', 'resistance training', 'aerobic training', 'training', 'intervention', 'randomized controlled trial', 'randomised controlled trial', 'effect', 'program' or 'outcome'.

\section{Participant or population: Adults.}

Intervention: Physical activity program (e.g., aerobic, resistance, concurrent).

\section{Comparator: Control group without intervention.}

Study designs to be included: Randomized controlled trials.

Eligibility criteria: To be included in the meta-analysis, studies needed to meet the following criteria: (i) intervention: physical activity program (e.g., aerobic exercise, resistance exercise, multicomponent program or similar); (ii) participants: subjects over the age of 18 years; (iii) outcomes analyzed: myosteatosis (e.g., intramuscular adipose tissue, muscle attenuation or similar); and (iv) study design: RCTs.

Information sources: Two authors independently will search MEDLINE, EMBASE and SPORTDiscus electronic databases.

Main outcome(s): Intermuscular adipose tissue and muscle attenuation.

\section{Additional outcome(s): None.}

Quality assessment / Risk of bias analysis: Two independent authors will assess the methodological quality of the selected studies with the PEDro scale.

Strategy of data synthesis: All analyses will be conduct using the random-effects inverse-variance model with the HartungKnapp-Sidik-Jonkman variance estimator based on DerSimonian-Laird estimate of tau and carried out using the STATA software (version 13.1; StataCorp, College Station, TX, USA) to calculate the standardized mean difference, which will be expressed as Hedges $g$ (Higgins JPT, n.d.). Heterogeneity across studies will be calculate using the inconsistency index (12), considering 12 values of $25 \%, 50 \%$, and $75 \%$ as low, moderate and high heterogeneity, respectively. Small-study effects biases will be assess using the extended Egger's test, and the presence of publication bias was investigated visually by funnel plots.

Subgroup analysis: The potential moderating effect of age at baseline or intervention duration (weeks) will be examine using meta-regression suing method of moments (DerSimonian and Laird method).

Sensibility analysis: Sensitivity analysis will be conduct to assess the robustness of the summary estimates in order to determine whether or not a particular study accounted for the heterogeneity. Thus, in order to examine the effects of each result from each study on the overall findings, results will be analyze with each study deleted from the model once.

Language: English.

Country(ies) involved: Chile; Spain.

Keywords: Exercise; intermuscular adipose tissue; muscle attenuation.

Contributions of each author:

Author 1 - Robinson Ramírez-Vélez.

Author 2 - Yasmin Ezzatvar.

Author 3 - Mikel Izquierdo.

Author 4 - Antonio García-Hermoso. 